\title{
Transference and Countertransference in EMDR Therapy
}

\author{
Olivier Piedfort-Marin \\ University of Lorraine, Metz, France \\ Institut Romand de Psychotraumatologie, Lausanne, Switzerland
}

\begin{abstract}
Just like any other psychotherapy method, eye movement desensitization and reprocessing (EMDR) should conceptualize the intersubjective phenomena that are active during EMDR therapy, especially in the treatment of complex cases. This article describes the concepts of transference and countertransference and how to integrate them in the Adaptive Information Processing (AIP) model. In this article, research on mirror neurons, the concept of action systems, and recent considerations on attachment theory for patients with disorganized attachment are incorporated into the concepts of transference and countertransference. Input from each of these theories is illustrated with a clinical vignette that depicts how the client's and the therapist's conscious and unconscious processes are intertwined and how they may affect the efficacy of EMDR therapy. We propose the countertransference-based interweave to release the AIP when countertransference issues block the process. Integrating knowledge on transference and countertransference in EMDR therapy could increase the efficacy of EMDR, especially in complex cases.
\end{abstract}

Keywords: transference; countertransference; EMDR; clinical practice; psychotherapy; therapeutic relationship

$\mathbf{E}$ ye movement desensitization and reprocessing (EMDR) is an integrative approach that requires the development of "a firm therapeutic alliance" (Shapiro, 2017, p. 114). Nevertheless, the means to develop such a firm therapeutic alliance or a good, constructive relationship between the clinician and the client has attracted little attention in the EMDR community. In the second and third editions of her manual Shapiro $(2001,2017)$ only devotes half a page to this topic. Dworkin (2005) has written a major contribution on the relational implications involved in EMDR. EMDR was first developed for the treatment of posttraumatic stress disorder (PTSD). The need for recognition of this new method has led to an impressive series of high-quality research. As in other methods, research needed for scientific approval fails to integrate the relationship between the clinician and the client and its possible impact on the efficacy of the method. What occurs between a client and the clinician is indeed subjective and intersubjective and can hardly be replicated. In clinical practice, clinicians need evidence-based methods but also tools to adjust such methods to the diverse clients they meet every hour. Furthermore, every clinician is a unique individual.
Since the early days of modern psychotherapy about 120 years ago, transference and countertransference have been an interesting way to understand patients' difficulties and pathologies. These concepts are considered central in several trauma therapies, and important in others, while they have attracted little attention until now in the EMDR community, to say the least. Pearlman and Saakvitne (1995) developed a complex but comprehensive handbook on the understanding and the use of countertransference in psychodynamic therapies of incest survivors. Kernberg and his team (Clarkin, Yeomans, \& Kernberg, 1999) consider transference central in the therapy of borderline personality disorders that are often trauma related. Dialectic behavioral therapy (DBT; Linehan, 1993) and schema therapy (Young, Koslo, \& Weishaar, 2003) also take into consideration the role of the relationship between the clinician and the client when treating borderline patients. In DBT, supervision plays a key role in supporting clinicians who often find themselves dealing with difficult moments with their clients. In cognitive behavioral therapy of PTSD, there is some limited consideration for phenomena in the client-therapist relationship that can be categorized under transference and countertransference 
(Brillon, 2005). In family therapy, resonance (Elkaïm, 2008) is a core concept and tool of change that is close to the concept of countertransference. It is difficult to believe that EMDR is the sole trauma therapy that does not need to take transference and countertransference into consideration.

When working with trauma patients, clinicianslike any human beings - do react, either consciously or unconsciously, to the terrible and sometimes horrific stories they hear in their office. How could it be otherwise? As Dworkin developed in detail in his book (2005; see also Dworkin \& Errebo, 2010), these conscious and unconscious reactions may have an impact on the client and on the relationship between the client and the clinician, thus impacting the use and efficacy of any chosen method, EMDR in our case. The Diagnostic and Statistical Manual of Mental Disorders (5th ed.; DSM-5; American Psychiatric Association, 2013) takes this into consideration when describing the types of exposure to trauma needed to meet criterion A of PTSD. It includes "Experiencing repeated or extreme exposure to aversive details of the traumatic event(s) (e.g., first responders collecting human remains and police officers repeatedly exposed to details of child abuse)." Psychologists and psychiatrists are indeed included in this type of exposure to trauma. Being a trauma therapist involves being confronted with stories of disturbing and sometimes horrific events, and it may have an effect on many therapists, including EMDR therapists (Schubbe, 2011).

This article proposes ways to understand how transference and countertransference can impact the use and efficacy of EMDR. We will describe how to integrate these originally psychodynamic concepts into the Adaptive Information Processing (AIP) model and give clinical examples to illustrate our views. This article supports the work of Dworkin (2005) and adds to his pioneer work inputs from the theory of mirror neurons, attachment theory (mainly the work of Liotti, 2011), and the concept of action-systems (Van der Hart, Nijenhuis, \& Steele, 2006). This way, transference and countertransference may be more accessible for those EMDR therapists who are not trained in psychodynamic therapy. Clinical vignettes will illustrate these theoretical perspectives.

\section{Transference and Countertransference}

\section{Brief History of the Concepts and Their Definition}

For quite some time now, the definition of transference has been commonly agreed on as "the unconscious repetition in a current relationship of patterns of thoughts, feelings, beliefs, expectations, and responses that originated in important early relationships" (Pearlman \& Saakvitne, 1995, p. 100). Originally, psychoanalysis focused on transference as a way to obtain access to the client's unconscious difficulties and conflicts. The concept of countertransference was first mentioned by Ferenczi (1916). Freud (1910) considered this to be a problem in analysis that should be neutralized and overcome by the therapist. According to Freud, analysis was supposed to focus solely on transference. Nevertheless, as he was going back to the traumatic origins of neurosis, Ferenczi (1988) considered that using countertransference gave new and rich possibilities to the analysis, especially when working with trauma patients. The definition and the use of countertransference has since been developed under the influential work of Heiman (1950) and Racker $(1957,1968)$. Hayes (2004) summarizes Freud's narrow definition of countertransference as "the unconscious, conflict based reactions (of the therapist) in response to the patient's transference." Later Heiman gave countertransference a broader definition, including all therapists' reactions to the client, not only unconscious but also conscious, not only in response to the client's transference but also in response to any other material. Both Heiman and Racker brought a radical change in psychoanalysis, considering countertransference as the main working tool of the analyst and also as a better way to explore the client's unconscious. A third phase of the development of the countertransference definition came from Gelso and Hayes (1998). Their focus was more on the unresolved conflicts of the therapist as the main source of his or her reactions to the clients, and therefore gave a key role to the clinician's responsibility in the intersubjective phenomena at stake during psychotherapy. This last definition, in line with Ferenczi's pioneer work, greatly influenced Dworkin's work in the use of countertransference in EMDR.

\section{Countertransference With Sexually Abused Clients}

Pearlman and Saakvitne (1995) made a major contribution by adjusting the concept of countertransference for therapies of sexually abused individuals, which can also be useful in EMDR therapy. According to these authors, countertransference has two main components: (a) "The affective, ideational, and physical responses a therapist has to her client, his clinical material, transference, and reenactments, and (b) the therapist's conscious and unconscious defenses against the affects, intrapsychic 
conflicts, and associations aroused by the former" (Pearlman \& Saakvitne, 1995, p. 23). Furthermore, they describe different factors contributing to countertransference in trauma therapies:

1. The therapist's response to the reality of incest and child abuse.

2. The therapist's responses to the client's transference, which will vary with both the nature of the transference and its consistency with her own experience of self.

3. The therapist's response to the client's particular posttrauma adaptation, for example, numbing, flooding, dissociation, intrusive imagery and memories, repression, anxiety, chronic suicidal wishes, depression, despair, interpersonal mistrust, revictimization, selfloathing, and so forth.

4. The therapist's history, personality, coping style, and transference to the client.

5. The therapist's response to her own vicarious traumatization.

6. The therapist's theoretical perspective on trauma and relationship to her teachers and mentors in the field. (Pearlman \& Saakvitne, 1995, pp. 24-25).

To the first point, we could add the therapist's response to the reality of neglect and relational trauma, and his theoretical perspective on these somehow new areas of research in the trauma field since Pearlman and Saakvitne wrote their book in 1995.

\section{The Use of Countertransference}

Pearlman and Saakvitne (1995) stated that in trauma therapies clinicians can use their countertransference to help clients become aware of previously unconscious or dissociated affects, thoughts, or conflicts. When aware of a specific sensation, affect, image, or thought that the clinician believes has to do with the client, he may use these elements in a verbal intervention that will help the client gain awareness of them and connect with these previously unconscious or dissociated elements. In other words, Pearlman and Saakvitne see countertransference more as an opportunity to understand underlying therapeutic processes, or blockings to be released when needed. Later in this article, several clinical vignettes will illustrate how this view is adapted to EMDR.

On the other hand, Dworkin (2005) and Dworkin and Errebo (2010) insists on the therapist's responsibility: The countertransference can influence the therapeutic process and the clinician can release it in taking responsibility of his/her countertransference. For example, the trauma processing of a client might be blocked because the therapist has himself not fully processed a similar issue. If he realizes this and takes responsibility for the blocking in an adapted verbal intervention, this may release the client's processing. Both views are useful in EMDR as well.

\section{Transference and Countertransference Defined for EMDR}

Dworkin, 2005 proposed a definition of transference and countertransference compatible with the AIP model of EMDR. He defines transference in EMDR as "the activation of a state-dependent memories directed toward the clinician" and countertransference as "the activation of state-dependent memories in the clinician that have been sparked by the client, intentionally or not." I propose a definition that is somewhat closer to the EMDR terminology. Transference can be considered as the activation of dysfunctionally stored (mainly trauma or problematic attachment) memories of the client in relation to the therapy, the clinician, or the relation to the clinician. Countertransference can be defined as the activation of dysfunctionally stored (mainly trauma or problematic attachment) memories of the therapist by the client, his history, his material, and his relation to the clinician, consciously or unconsciously.

\section{Recent Helpful Research Regarding Transference and Countertransference}

\section{Mirror Neurons}

Recent research can offer new perspectives on transference and countertransference. These phenomena have been observed for over a century but have not really been explained. For example, nobody knows how to explain why a particular image related to his client comes to the clinician's mind and the next moment the client is describing that same image. No one can explain why the clinician is suddenly having difficulty staying awake during a session with a particular client but not with the next client, 20 minutes later. For a scientific mind, these are mysteries that may be understood in the future or simply not taken into consideration because they are not measurable. Such phenomena are indeed disturbing. The theory of mirror neurons might give a possible scientific explanation of at least some of the (EMDR) therapists' countertransference experiences. Rizzolatti, Fadiga, Gallese, and 
Fogassi (1996) discovered by accident that the same neurons are activated in rhesus macaques when the animal makes an intentional movement of taking a banana to eat it and when the animal sees a scientist taking the banana, and also when it hears related sounds (Kohler et al., 2002). Even though the role of mirror neurons in humans is subject to critique (Hickok, 2009), a similar system seems to be present in humans (i.e., Pizzamiglio et al., 2005). There seems to be a link between empathy and the activation of mirror neurons, as shown in a study on humans by Gazzola, Aziz-Zadeh, and Keysers (2006). Mirror neurons could be implicated in the ability to predict another person's action (Wilson $\&$ Knoblich, 2005), a phenomenon linked to empathy and attunement. According to Gallese (2009; also Gallese, Eagle, \& Migone, 2007), mirror neurons may also play a role in the sharing and transmission between human beings of implicit, sensorimotor, and prelinguistic material such as in transference and countertransference.

In EMDR therapy, client and clinician sit close to each other and target very sensitive material. The clinician, especially if using eye movements as bilateral stimulations (BLS), will look at the eyes of the client more closely than in other therapy approaches. Some of the clinicians' psychological processes could be explained by the concept of mirror neurons as shown in the following vignette.

\section{Clinical Vignette}

While using EMDR with a client to reprocess a memory of neglect, using eye movements as BLS, the therapist suddenly felt sleepy. It even became very difficult for him physically to continue moving his arm from left to right in front of the client's eyes. His arm started to shake. His eyelids started to close. He did not understand what was happening since the EMDR therapy with this client had being going on well over quite a long period of time. The clinician started to focus on his library in front of him for a few seconds, instead of on his client's face. When he focused on his books, the sleepiness vanished immediately. When he looked at his patient again, all of a sudden he felt sleepy again. He did this test several times with the same result. In this case, we can hypothesize that a mirror neuron effect occurred. While looking into the eyes of his client the clinician felt sleepy, but his physical reaction was completely the opposite when he looked at the books on his bookshelf. After the session, he felt disturbed but not only by the fact that he had almost fallen asleep during an EMDR session. He decided to do EMDR on himself using tactile pulsers as BLS, simply concentrating on the client and this last session. After a few sets of BLS, an affect bridge occurred, activating a traumatic event of the clinician. This event had been reprocessed with EMDR a few years earlier, successfully so he thought, but it was suddenly triggered with intense emotion and strong body sensations. His score on the subjective units of disturbance (SUD) scale was 9 (on a scale from 0 to 10,10 being the highest disturbance). This event had similarities to a traumatic event the client had experienced although it was not the same event that they were working on that day. Going back to therapy to resolve this issue, the therapist realized that he had unconsciously wanted to avoid working with this particular client because it activated a trauma memory that he was not ready yet to fully reprocess. This vignette illustrates how unconscious material can be communicated between two human beings and how this may impact the therapeutic process of EMDR.

\section{Action Systems and Subsystems}

\section{Definition of Action Systems and Subsystems}

The concept of action systems and subsystems was developed by Van der Hart et al. (2006), influenced among others by Panksepp (1998). Developed in the context of the theory of structural dissociation of the personality Van der Hart et al. (2006), the concept of action system can also be useful for noticing and analyzing the client's and the therapist's reactions during sessions. It may be helpful in analyzing transference and countertransference in a somewhat more structured manner, which can be useful for therapists, especially those not trained in psychodynamic therapy. Van der Hart et al. (2006, p. 3) defined action systems as "psychobiological systems that make up personality" and involve specific "innate propensities to actin a goal directed manner" (emphasis added). There are two main categories of action systems, each of them consisting of subsystems with more specific goals. The first category of action system implies approaching attractive stimuli and dealing with daily life. Nutrition, exploration, reproduction, caregiving, social engagement, and social ranking are some of the action subsystems included in this category. The second category of action system implies the avoidance of adverse stimuli and coping with threat and danger, with such action subsystems as hypervigilance, fight, flight, freeze, and submission. 
Action Subsystems as a Tool to Analyze

Transference and Countertransference

Clinicians can evaluate action subsystems used by clients during sessions by observing their behavior, speech, and sensorimotor actions. These are useful to become aware of the transference in a structured manner, especially for clients with complex posttraumatic stress disorder (C-PTSD) and dissociative disorders (Piedfort-Marin, Wisler, Spagnoli, \& Piot, 2017).

The observation of action systems and subsystems activated in the clinician can also be a useful structured way to analyze countertransferential reactions of clinicians in treating trauma patients (Ellert Nijenhuis, personal communication, 2008-2016). If a clinician bends over and leans toward the client, his or her attachment or caregiving subsystems might be activated. If a clinician holds back and feels stomach tension, the defense action system might be activated. When clients have a history of (possibly repetitive) physical and/ or sexual violence, they have learned that significant others can change and suddenly become aggressive or disconnected. As a result, they tend to be careful and notice such sensorimotor actions even before the clinician becomes aware of them. Therefore, it could be useful for clinicians to become aware of their own countertransferential reactions as soon as possible, so that they can adjust their interventions.

\section{Analysis of Action Systems and Subsystems in EMDR}

Here are the main action subsystems clinicians can feel when working with trauma patients in EMDR.

\section{Defense Action Subsystems During EMDR Therapy.}

- Avoidance-flight: That is, avoiding listening to the client's story and traumas, therefore avoiding doing trauma processing (Phases 3-6 of EMDR treatment), or doing trauma processing as soon as possible to get it over with as fast as possible.

- Submission: That is, obeying the client's demand to do EMDR no matter what the conditions are, or obeying the therapist's EMDR mentor/consultant/trainer without reflecting the specific situation that the clinician and the client are engaged in.

- Aggression: That is, reacting with irritation or frustration when a patient doesn't elaborate the negative or positive cognition or when he/she is not processing the way the clinicians believe he/ she should. This may stress some clients to such an extent that their difficulties to find a cognition increase (Dworkin \& Errebo, 2010).
Action Subsystems Directed Toward a Stimulus During EMDR Therapy.

- Attachment: That is, when a clinician feels attached to the client.

- Social ranking/domination: When a clinician, seeing himself as a specialist, feels the need to be dominant toward the client. This can occur when the clinician imposes his/her view of how the therapy should be. In numerous species, this action subsystem sustains the organization of a group of individuals in a way that is useful for the survival of the species. Nevertheless, EMDR therapy is considered as a client-centered approach (Shapiro, 2001); therefore, collaboration should be enhanced and not social ranking.

- Play: A certain dose of play can be useful in psychotherapy. Nevertheless, overuse of this subsystem could be a sign of avoidance on the part of the therapist or of both therapist and client.

- Exploration: The ability to discover and explore the unknown; for example, to explore symptoms by trying to understand them.

- Social engagement/collaboration: The ability to engage in a healthy collaborative relationship, without an excessive need of attachment or domination.

- Cooperation: The ability of two or more individuals to work on a task with a common and shared goal.

Social engagement and cooperation are optimal action subsystems in EMDR. EMDR is defined as a client-centered approach (Shapiro, 2001); therefore, the subsystems of social engagement, exploration and cooperation should have priority in EMDR psychotherapy.

By observing and analyzing their thoughts, emotions, or sensorimotor actions or reactions, clinicians can then define which action subsystem is activated. They may first be unaware of the aim behind the activated action subsystem, but increased awareness will allow for a better understanding of the rationale behind the activated action subsystem. They can then adjust their intervention in accordance as we will see later in this article. Therapists can ultimately resolve their underlying issue and bring forward action subsystems that are optimal for EMDR therapy, social engagement, exploration, and cooperation.

Referring to action subsystems can be a useful structured manner to analyze countertransference, especially for clinicians who are trained in other approaches than psychodynamic. 


\section{The Implication of Disorganized Attachment on the Therapeutic Relationship}

\section{Disorganized Attachment as an Early Relational Trauma}

Liotti's work (i.e., Liotti, 2011) is helpful in understanding the impact of the client's disorganized attachment style (DA) in the client-clinician relationship and also subtle but meaningful phenomena in EMDR therapy. DA is overrepresented (from $40 \%$ to $80 \%$ ) in children of high-risk samples such as clinical groups, families with violence, or emotional disorders (Liotti, 2013; Lyons-Ruth \& Jacobvitz, 2008). It is hypothesized that DA develops when the child is facing a caregiver who cannot comfort the child properly or who is simultaneously a source of danger and a potentially comforting figure (Main \& Solomon, 1990). A large number of studies have shown that DA in infancy and childhood seems to be a risk factor in the development of psychological disorders in adulthood where deficit in integrative functions and in mentalizing abilities are involved; disorders are also linked with childhood traumas and adverse experiences (Bateman \& Fonagy, 2004; Dozier, Stovall-McClough, \& Albus, 2008; Levy, 2005; Liotti, 2006; Liotti \& Gumley, 2008; Lyons-Ruth, 2003). On the other hand, it is well established that adult patients with C-PTSD, borderline personality disorder, or dissociative disorders show a high risk of having a disorganized pattern of attachment (Bakermans-Kranenburg \& van IJzendoorn, 2009; LyonsRuth, Dutra, Schuder, \& Bianchi, 2006; Riggs et al., 2007; Schmahl, Lanius, Pain, \& Vermetten, 2010; Stovall-McClough \& Cloitre, 2006; van Ijzendoorn \& Bakermans-Kranenburg, 2008). In fact, DA may be understood as an early relational trauma (Schore, 2009). But the pattern of DA in adulthood might not always be as clinically obvious (overt) as it is, for example, with severe borderline patients; it can also be covert in some individuals who developed strategies in the course of their early development to deal with the overwhelming emotions related to DA.

\section{Disorganized Attachment and Controlling Strategies}

DA is observed in children at 18 months of age in the Strange Situation Procedure (Ainsworth, Blehar, Waters, \& Wall, 1978). Before reaching school age, DA seems to disappear. Liotti (2014) hypothesizes that the unbearable and painful experience of the activation of both attachment and defense mechanisms forces the child to solve the possible fragmentation of the self by developing controlling strategies. These controlling strategies aim at developing a more organized behavior, bringing more stability in the relationship to the caregiver. The two main types of controlling strategies are (a) the controlling-punitive strategy, identified by aggressive behaviors toward the caregivers (attempts to punish or embarrass them), and (b) the controlling-caregiving strategy, identified by a caring behavior toward the caregivers, or inversed attachment (Liotti, 2011; Lyons-Ruth \& Jacobvitz, 2008).

Children with DA are unable to synthesize representations of self and others. They develop compartmentalized representations that "will hamper the integrative functions of consciousness, so that dissociative experiences and mentalization deficits, concurrent with the activation of attachment needs, are a likely consequence" (Liotti, 2013). The longitudinal study by Dutra, Bureau, Holmes, Lyubchik, and Lyons-Ruth (2009) confirms this theory by showing that children with DA in infancy develop more dissociative processes measured by the DES (Bernstein, Putnam, \& Putman, 1986) in late adolescence than children with organized attachment in infancy.

In some adult individuals, controlling strategies may stay stable over time and DA remains covered. In other adult individuals facing separations, losses, or other events that activate past relational trauma, and when compensatory systems are overpowered, the controlling strategies may collapse and the DA emerges (Liotti, 2014).

With adult clients with a-covered or overt-DA, Liotti $(2011,2013,2014)$ advises not to activate the attachment subsystem but to promote the cooperation subsystem instead. If the therapist is too close or too distant, this may activate early relational trauma memories and brings up what Liotti calls "a rupture of the therapeutic alliance." Under the activation of the relational trauma memories, the client will engage in controlling strategies with the therapist (transference), either caregiving or punitive, and this may change the course of the therapy by its effect on the therapist's countertransference.

\section{Disorganized Attachment and Cortical Connectivity}

An interesting study by Farina and colleagues (2013), in which Liotti participated, tested the activation of relational trauma memories on the cortical connectivity in two groups of adult subjects: 13 patients with DA and C-PTSD or dissociative disorders, and 13 healthy control subjects with an organized 
attachment. Farina and colleagues (2013) state that "dynamic cortical connectivity networks are considered to play a crucial role in high-level cognitive functions: working memory, top-down executive functions, attentive tasks, and consciousness." Therefore, the authors measured cortical connectivity "as an index of integrative and disintegrative processes" at stake while activating relational memories with the Adult Attachment Interview (AAI; George, Kaplan, \& Main, 1996). The modifications of cortical connectivity (before and after the AAI) were measured by electroencephalography (EEG) and lagged coherence analysis. These measures showed a widespread increase of cortical connectivity in the control group but no increase at all in the DA group. After the AAI was performed, there was an increase of sympathetic activity in the DA group but not in the control group.

To summarize the conclusions by Farina and colleagues, when relational memories are activated (by the AAI), individuals with an organized attachment react with emotions and sensations and their mentalization processes are also activated (increase of cortical connectivity), so that they can feel the emotions and regulate them. It is just the opposite in individuals with DA: the mentalization process will not activate (no increase of cortical connectivity) and disconnection is the only way to deal with the emerging disturbing effects. Indeed, relational memories are often traumatic in individuals with DA and C-PTSD or dissociative disorders as in this patients group. The following vignette illustrates how this study can be useful to EMDR therapists.

\section{Clinical Vignette}

Mrs. A. is a 40-year-old woman with comorbidities that can be understood as the consequence of complex traumatization (child neglect, family dysfunction, early death of her mother, and sexual abuse): severe eating disorder with obesity, depression, self-esteem issues, and difficulties at work. She has been in treatment with her therapist for 3 years with a focus on EMDR, which works well with her. One day her husband leaves a message at the therapist's office, stating that she had been hospitalized and will not be able to come to her session for 2 weeks. Worried and wondering what has happened, the therapist calls her husband to know more about what has occurred: The client had needed immediate surgery related to a pancreas infection. When the client comes back to therapy, her health is good and the psychotherapy can carry on. They agree to continue the work on the situation of neglect, which they were working on before the hospitalization. The therapist asks the client to focus on the target situation and the client is surprised to state that nothing comes up and that the SUD is 0 . "There should be something coming up, I know we haven't reprocessed all of this situation," she said. Despite her best efforts, she is unable to see or feel anything disturbing when focusing on this same situation that had brought her noticeable and painful thoughts, images, and affects in the session prior to her surgery. The therapist then asks her if she can explain this or if anything has happened since the last session or during her hospitalization. "The only thing that has happened is that you called to have news about me and my health. You cared for me and no one ever did when I was a child," said the client with tears. The rest of the session was spent on further elaboration on the effect of the therapist's telephone call. When she is asked to focus again on the target situation in the following session, she is able to notice disturbing thoughts and emotions and a related SUD and the reprocessing is back on track.

The therapist triggered traumatic relational memories of neglect when he called the client's husband to get information on her health. Even though the attachment style of this client had not been measured, neither in infancy nor in adulthood, from the information collected, it is most probable that the client had a DA in infancy and developed controlling-punitive strategies with her mother a few years later, and later in life controlling-caregiving strategies with her husband and her therapist. While she could always connect with the disturbing emotions of several traumatic or highly disturbing memories with the EMDR protocol and in other ways, this time she was somehow disconnected from the relational traumatic memory. Liotti (2013) calls this phenomenon a dissociative process, with detachment and compartmentalization. I understand that the cortical connectivity of the client didn't increase when the memory of a relational trauma of neglect was triggered again with the EMDR protocol. This is why a SUD of pseudo 0 was measured. In all previous and later sessions with this client, the therapist had a more neutral attitude, activating the cooperative action subsystem, which allowed for a good trauma processing (and a probable increase of cortical connectivity). Nevertheless, the length of her therapy may be explained by a certain lack of mentalization abilities that existed but were not made obvious until this incident. 


\section{Implication for EMDR Therapy}

During trauma processing (Phase 4), the AIP can be blocked when the patient lacks sufficient emotional regulation abilities to integrate a dysfunctionally stored memory, that is, when dissociative processes are too strong. But processing can also be blocked solely by the clinician's attitude. If, by their attitudes, clinicians trigger relational trauma memories of patients with complex trauma-related disorders, the integrative processing required for a successful EMDR therapy may be blocked, independently from the content of the targeted memory. EMDR practitioners should not be too close nor too distant when working with clients who may possibly have DA (whether overt or covert). In line with Liotti (2011), it is important to keep a cooperative attitude when working with clients with C-PTSD or dissociative disorders of any kind or any disorder that implies a possible DA. In the previous vignette, the client was well aware of her difficulties and the rupture of the relationship could be repaired easily. This may not always be the case. When EMDR clinicians are too close (caregiving action subsystem) or too distant (social ranking/domination action subsystem or defense action system), their attitude may activate the client's relational trauma memories. The integrative cognitive and affective process needed to neutralize the client's trauma memory through AIP model will not be activated (as a measure of cortical connectivity could probably show, according to the study by Farina et al. [2013]). A pseudolow SUD may occur and some clinicians may falsely believe that the memory is resolved and not disturbing anymore.

\section{Transference and Countertransference in the Different Phases of the EMDR Protocol}

EMDR therapy is based on an eight-phase protocol. Phase 1 (client history and treatment planning) and Phase 2 (preparation) are not unique to EMDR therapy, with the exception of the information about EMDR that is given to the client in Phase 2 immediately prior to Phase 3 . In fact, other trauma therapies advise to proceed by a client history, a treatment planning, then stabilization before trauma confrontation, according to the guidelines of the International Society for the Study of Trauma and Dissociation (2011) and the ISTSS (Cloitre et al., 2012). Phases 3-6 are specific to EMDR and follow a structured and guided protocol. Phases 7 and 8 address, respectively, the closure of a session and the reevaluation of the previous session; they are of lesser interest in regard to the aim of this article. We will present the specificities of transference and first for Phases 1 and 2, then for Phases 3-6.

\section{Transference and Countertransference in Phases 1 and 2}

In Phase 1, the clinician welcomes the client and starts by taking the client's history, then does an evaluation of safety factors to determine whether the client is suitable for EMDR therapy, before deciding upon a treatment plan. In Phase 2, the clinician establishes a therapeutic alliance, gives information on trauma processing with EMDR, and introduces safety procedure and relaxation exercises. In these two phases, EMDR therapists have the difficult task of deciding whether trauma processing is indicated at the present time or if more time is needed to establish a suitable therapeutic alliance and/or affect regulation procedures. Another task is to set up a treatment plan. In these phases, clinicians' decisions are not only dependent on objective observations and facts but also on several subjective factors that are listed here, in line with Pearlman and Saakvitne (1995) described previously.

1. The therapist's response to the reality of the client's specific traumas: How severe does the EMDR therapist consider that particular trauma to be? Does the EMDR therapist consider, for example, neglect to be a core issue in the development of pathology?

2. The therapist's responses to the client's transference: That is, can the clinician resist some clients' pressure to begin trauma processing very quickly in the therapy without taking into account or assessing possible safety issues? How does the EMDR therapist respond to a client who puts him on a pedestal because he uses EMDR?

3. The therapist's history, personality, coping style, and transference to the client. Has the therapist experienced a traumatic experience similar to the client's? How does it affect the assessment of the patient and the treatment planning?

4. The therapist's response to the activation of her own traumas by the client or the client's history: Will the EMDR therapist avoid the activation of her own traumas; for example, by extending Phases 1 and 2 more than needed, or by reducing the necessary time for these two phases?

5. The therapist's response to her own vicarious traumatization that may immediately occur during history taking. Here again avoidance may be a reaction on the part of the EMDR therapist.

6. The therapist's theoretical perspective on trauma and relationship to her EMDR trainers and EMDR 
consultants. The position of the therapist's mentor on the stabilization controversy can have an impact on her decisions, depending on the relationship she engages in with the mentor.

During Phases 1 and 2, the EMDR therapists' traumas and attachment issues can have an impact on the way they understand and conceptualize the clients' cases and how they set up the treatment plan. Some therapists may not be aware of such an influence on their clinical judgments and choices. When clinicians are in therapy to seek treatment for their own posttraumatic symptoms or attachment issues, this may also interfere with their clinical choices. EMDR therapy may be difficult or blocked when a therapist works on similar issues or similar traumatizing events in his or her own therapy as the client. In such cases both client and therapist are activated and it requires tremendous energy for the therapist to maintain his working abilities, a proper distance, and due empathy toward the client. The client may feel that something is going on and may feel responsible. Here is a clinical vignette that illustrates this.

Clinical Vignette: When the Therapist Is Also in Therapy

Mr. C., a 30-year-old social worker, comes to therapy for sexual difficulties due to several sexual assaults he experienced when he was 3 years old. The parents brought him to their family doctor to ask for advice and support but this consultation was of no help, since the doctor banalized such events. After nine sessions for Phases 1 and 2, with a thorough history taking and the learning of safety procedures, the clinician considered that this was a noncomplex traumatization and proceeded to trauma work with Phases 3 and 4 of the EMDR protocol. After eight sessions and a significant decrease of the SUD, the client called the therapist to tell him that pedophile ideas had emerged after the last session. Safety procedures were put in place in real life since the client was sometimes working with children and adolescents. Stabilization exercises were used to decrease these new symptoms and the high disturbance they had on the client. The therapist was astonished to feel insecure in his ability to work with the stabilization exercises although he was well trained and experienced in them. He felt that his working abilities were diminished "for no reason." A consultation revealed that he had not properly assessed the emotional neglect the patient had experienced in the relationship with his parents and the negative effect this had on the resilience factors. So he was himself neglecting the client in the same way that the client's parents did, by not taking fully into consideration the severity of the sexual assaults. During this consultation, it became clear to the therapist that he disliked some controlling behaviors of the client that reminded him of his controlling mother. This caused him to be distant to the client, another type of neglect. Furthermore, he realized that he was himself working on some type of sexual abuse in his own therapy. In fact, he had his own weekly therapy session on the same day as his client's session. It took tremendous energy for him to separate his own issues from his client's issues and to fight his own reactivation while working with his client. The whole situation had blocked the therapy. The consultation allowed for a clearer diagnostic and for an adaptation of the treatment plan. The therapist took responsibility for his own role in the difficulties occurring during the therapy, addressed the controlling tendencies of the client with empathy, and explained the needed adjustment of the treatment, which the client understood and accepted.

\section{When Clients Want Immediate Trauma Processing}

Among clients who wish to undertake EMDR therapy, some of them expect it to be magical. I am always amazed by clients who expect EMDR therapy to last a maximum of four sessions (with Phases 1 and 2 included), while research has proven that the efficacy of EMDR uses a nine-session format for the treatment of "simple" PTSD. I am even more surprised in consultation that colleagues-even experienced ones-follow the expectations of such clients and start with Phases 3 and 4 on the second session with patients who have experienced complex traumatization. It is not the aim of this article to discuss the controversy of whether trauma processing should start very early in therapy of clients with C-PTSD or dissociative disorders. Nevertheless, focusing on the subjective and intersubjective factors possibly involved in the treatment planning of EMDR therapy may help to articulate aspects of the controversy.

Individuals who experienced complex traumatization in childhood and/or adolescence may come to EMDR therapy expecting fast results. This is fully understandable since they may have a long history of suffering and of nonefficient or partially efficient therapies. Everyone is entitled to happiness 
or at least inner peace. Such patients have often experienced specific traumas (physical or sexual violence, or even a car accident) as well as neglect. For example, their parents or caregivers may not have comforted them after an injury or may have screamed at them for being not careful enough after an accident. Some parents may be technically good parents: for example, they would take the child to a psychotherapist after the child has been sexually assaulted, but they may not be able to comfort the child. These are technically good but emotionally neglectful parents. A relational trauma (lack of comfort or shaming) is added to the actual trauma (the abuse). EMDR therapists should be careful while investigating the expectations of some clients with C-PTSD who consider EMDR as a fast and technical therapy. Some of them may transfer their own relational trauma onto the method and onto the clinician. Behind an entitled need of a fast therapy ("I have suffered enough, I want a fast and technical therapy that has been proven efficient"), EMDR practitioners should be aware of a possible unspoken belief such as "my parents never took any time to listen to me, so I don't expect you or anyone else to take any time to listen carefully to me, so let's get started with the technical stuff." If the clinician decides to follow the expectation of the client and start early with trauma processing (Phases 3-6), he will reactivate the relational trauma of the client: not being listened to, not being sufficiently taken care of. The clinician will repeat the client's parents' behavior: technically good but emotionally neglectful. This could reinforce a negative cognition like "I don't deserve to be listened to" or "I am not worthwhile."

We have understood since Ferenczi (1949) that some individuals with a history of early abuses reenact their early traumas in therapy. EMDR therapists should be careful not to participate in such reenactments but to find out what their client's expectations are concerning trauma processing with EMDR. Clinicians should also analyze their own motivation for agreeing with the patients' request for a fast therapy or of rapid trauma processing or for refusing it.

\section{When EMDR Therapists Go Too Quickly to Trauma Processing}

We observe several pitfalls when the client or his/ her life and issues are activating past trauma or inner conflicts on the side of the clinician. Some EMDR therapists may want to go quickly to trauma processing, without being conscious of their inner motivation to get it over with as quickly as possible. Others may not fully assess all aspects of the client's traumatization, in some cases because they unconsciously avoid the same issue in themselves. Others can be afraid to start trauma work and so they extend the stabilization phase with extra safety procedures. The action subsystem of avoidance/flight is activated within the therapist in all these cases.

The idea of working on certain specific traumatic events (e.g., sexual violence or certain type of accident) may provoke feelings of sadness, fear, anger, despair, or disgust in some therapists. Trauma processing may be impaired if the therapist is not (yet) able to stand by the client's side in such a difficult journey. Clients may feel unsafe if they feel or realize that the therapists are concerned about their own fears and cannot support them enough. This may activate memories of past unsafety and disturb the trauma work that is processed. Therefore, in this case it might be better for the client not to proceed to trauma processing if the EMDR therapist doesn't feel ready (yet) to do it. Such issues are not due to a wrong assessment of the client's situation but to the therapist's own feeling of unsafety toward trauma processing in general or in specific cases.

The following vignette focus on the implication of starting trauma processing too early. We will see how the therapists' own trauma can be involved in the decision to start trauma processing too soon.

\section{Clinical Vignette: Early Trauma Processing as a Way to Avoid the Own Trauma of the Therapist}

Mrs. B. is an experienced EMDR practitioner and psychotherapist. She comes to my consultation with the following question concerning a new patient: "He dissociates during reprocessing; what should I do?" I didn't answer this question immediately and asked for more information about the client. It appears that this man was born in a former Soviet country until his family moved to Switzerland in the beginning of the 1980s where he and his parents regularly experienced humiliating situations due to their status as migrants. His father was physically violent with him and put extreme pressure on him to succeed. His symptoms can be categorized as C-PTSD with comorbidities. The client, who earns a good income, demands a short EMDR treatment of four to five sessions. The clinician warned the patient that this would be difficult but still did Phases 3 and 4 in the second session after a short safe place exercise that was only partially satisfactory. While she was relating some details 
concerning the client's life, the colleague realized that she was emotionally activated. After reflecting, she realizes that her mother came from the same region as her client, and that she had been a migrant as well. Immediately afterward, humiliating and dangerous situations her mother had experienced during her migration through Europe during World War II came to her mind. With tears in her eyes, the colleague expressed her surprise that her client came back after the first session and then again after the second session. As we see, the problem was not that the client dissociated during Phase 4. The problem was that both client and clinician were activated and both wanted to get it over with as soon as possible. Both of them tended to avoid their own traumatic memories. In his desire for a short technical therapy, the client is probably reenacting the neglect and pressure to succeed that his father imposed on him (introjection). In her will to follow the client in doing a very brief therapy, the clinician is answering her own need to avoid her past traumas, activated by the origin of the client, his life as a migrant, and the experience of humiliation. Not just the client but both clinician and client were emotionally disconnected during Phase 4. After this consultation, the clinician took some time to renegotiate the frame of the therapy and properly investigate her client's history, taking into consideration her own possible activation of family traumas. Although she was aware of these traumatic events in her family history and had worked on them in the past, the clinician had not understood-until this consultation-their meaning in the work with this particular patient.

In my observations, traumatic situations can be activated within the clinician even though they have been successfully processed in the past. The depth of the trauma process could also be argued. A possible remaining print of the former dysfunctionally stored memory may suddenly be activated and exacerbated with a related increased disturbance. This may possibly be due to the effect of the mirror neurons and to the very unique setting of the psychotherapy.

As we see with these clinical vignettes, the assessment of the client's difficulties and resources and the setup of a treatment plan (Phases 1 and 2) are not simply dependent on objective data (if such things exist) but also on the sometimes complex subjective and intersubjective elements of the encounter between the client and the therapist. It can be useful to be aware of transference and countertransference phenomena that are active since the very first session. This may increase the efficacy of the treatment and make therapy safer for both clients and therapists.
Transference and Countertransference During Phases 3-6

Dworkin (2005) as well as Dworkin and Errebo (2010) described in detail the effect of transference and countertransference during all phases of the EMDR protocol. Their contributions are very helpful for a precise work on this core part of EMDR psychotherapy, using countertransference at its best to increase the efficacy of EMDR. While this article supports Dworkin's work, we will add some specific interventions that can also be of interest.

\section{Countertransference-Based Interweave}

Dworkin (2005) describes the relational interweave as a specific intervention when the processing is blocked due to a countertransference issue. He states ( $p$. 170) that "a relational interweave is called for when a state-dependent memory has been activated in the clinician, is noticed by the client on some level, and appears to have temporarily stalled the work." I propose another type of interweave also based on countertransference, which I call countertransference-based interweave. In some clinical situations, mainly during Phase 4, when there is a blocking, the therapist may have a specific action subsystem activated, and this may help understand the blocking in the client's processing. The analysis of the action subsystem activated in the therapist allows for an adapted intervention in the form of an interweave. The following clinical vignette will illustrate this type of interweave.

\section{Clinical Vignette}

Mr. D. came to therapy to treat a minor social phobia. It appeared that this was due-among other factorsto physical violence during childhood inflicted by the parents' employees. The parents had never been aware of this. While working on a scene of such violence, during Phase 4, the client is on the verge of an abreaction, as if he needed to cry strongly. Nevertheless, the abreaction doesn't come out, despite the usual procedures (longer sets of BLS, change of direction of the eye movements, change of type of BLS, and several classical cognitive interweaves) and this lasted for four sessions. During the fourth session, the therapist is surprised to feel an urge to take the client in his arms. He made the hypothesis that the therapeutic relationship is involved in the blocking and addresses this after interrupting Phase 4, using cognitive tools to analyze this issue. This didn't contribute anything new to the therapy. He then analyzed the situation 
from the perspective of action subsystems. The urge to take the client in his arms corresponds to the caregiving subsystem. The therapist concluded that this may be a sign of the client's need. In the next session, as the client was again on the verge of an abreaction during Phase 4 of the EMDR protocol, the therapist proposed the following interweave: "Could it be that the little boy needed to be held and comforted?" The client answered a loud "yes!" and busted out crying. The AIP was released and the EMDR therapy could continue with success.

This interweave was based on the therapist's sensation and sensorimotor reaction during the trauma processing. It is the awareness of the countertransference, analyzed with the concept of action subsystems, that made this interweave possible. This is why I call it countertransference-based interweave.

\section{Considerations on Countertransference-Based Interweaves}

In other situations, therapists may notice images, thoughts, feelings, or sensations in their own inner world that are related to the client or to the traumatic event that is targeted with the EMDR protocol. With Pearlman and Saakvitne (1995) I propose that this information may contain elements from which the client is dissociated. The mirror neurons may explain why some therapists "receive" such elements about their clients and/or the targeted traumatizing event. In many cases, such countertransference phenomena appear in conjunction with a blocking of the AIP. Indeed, if the client doesn't have conscious access to important elements of the trauma, the processing cannot be completed, at least not fully. The therapist has the choice of a countertransference-based interweave here as well. For example, while working with a client on a situation of sexual assault, if the therapist has the sensation of something on his leg, a possible interweave could be: "How is your leg?" or, more precisely, if the first proposition didn't release the AIP: "Could you possibly have some sort of sensation on your leg?" If again such interweaves have no success in releasing the AIP, then a more direct intervention can be tried out: "I feel something on my leg." Roques (2004) proposes the following intervention: "You transmitted me something" (then the therapist depicts the element to the client), in this example "You transmitted me the feeling of something on the leg." I consider this type of interweave too directive and even abusive in some way. As therapists, we do not know for sure why and how we "receive" images, thoughts, affects, or sensations that we believe may come from our client. Therefore, it is imperative that a countertransference-based interweave be made in the form of a question, a proposition, or that it depicts strictly our own subjective experience.

Before making a countertransference-based interweave, therapists should analyze if the elements activated are (a) the therapists' own material, or solely (b) some of the client's dissociated material. In the first case, we recommend Dworkin's relational interweave (2005). In the second case, the countertransference-based interweave depicted here gives a good chance of releasing the AIP. Therapists may be disturbed when they "receive" such material. Indeed, some material is disturbing. Nevertheless, they should see this as a sign of a solid therapeutic collaborative relationship with their client. Safety procedures may help therapists in such situations: They may imagine themselves in their safe place while working (Dworkin, 2005), or they may practice meditation and take a more distanced position while working with their clients, or at least some of them. Taking a position of observer (Piedfort-Marin \& Reddemann, 2016; Reddemann, 2011) may help therapists to be close to the emerging material and at the same time emotionally distant enough for a more comfortable work.

\section{Discussion}

This article proposed that EMDR psychotherapy, like any other psychotherapy, should also focus on possible transference and countertransference issues to increase its efficacy, especially in the treatment of complex cases. Working with countertransference in EMDR therapy is also possible for therapists who are not trained in psychodynamic therapy or psychoanalysis and who wish to develop observation and analytical tools.

To notice countertransference, the best tool is to develop auto-observation abilities, awareness, and mindfulness. To become mindful of what is occurring in the relationship with clients and in oneself about a client requires some training. Some therapists may be more capable than others of being mindful but this is a quality that can be developed in different ways. Some may benefit from mindfulness training, others from meditation, and others may have their own way of becoming more mindful. Developing an "inner observer" may be an interesting tool as well. It can be useful for therapists to concentrate on different levels of information: what the patient says and shows, but also what the therapist feels, thinks, does, and "sees" about the client, about himself, and about their relationship. The therapist should also stay focused on what he doesn't feel, think, nor do. Any change in his working habits could be a sign 
of a possible countertransference issue. Realization of a countertransference issue can emerge at any time: at home, at sport, in a dream, while with another client, and so forth. Consultation is a specific space to allow the emergence of possible countertransference issues with the empathic help of the consultant. Often a therapist feels that "there is something going on" but will need a consultation to realize what the problem is. Consultants can help therapists develop their observation skills in regards to countertransference.

The understanding of countertransference issues may benefit from a structured analysis. A basic toolespecially in trauma therapy-is Karpman's dramatic triangle (Karpman, 1968): Therapists should notice whether they are in the role of the victim, the rescuer, or the persecutor, and should make a conscious effort to stay out of any of these positions. Another triangle presents the hurt child, the molester, and the nonprotecting caregiver. This last position is sometimes replaced by the technically good but emotionally neglectful caregiver, a position that can occur more often in "technical" therapies like EMDR. This is why EMDR therapists should be aware of this possible trap. As (Watkins \& Watkins, 1997, p. 99) stated: "A general principle, perhaps in all therapy, is the following dictum: Don't do what the parents, or whoever reared the patient early in life, did wrong in the eyes of the patient, whether it is ignoring or abuse." Finally, the concept of action systems and subsystems (Nijenhuis, 2017; Van der Hart et al., 2006) is an interesting tool that allows for a structured analysis of the therapist's thoughts, emotions, images, and sensorimotor actions and reactions. This tool may be better integrated by therapists with a cognitive-behavioral background.

It may also be important to analyze the conscious and unconscious motivations that brought clinicians to train in a particular therapy. Therefore, EMDR therapists should ask themselves the following basic question: Why did I choose to train in EMDR in the first place? The answer may reveal specific needs of therapists, such as the need for success, the need to be in control, the need to be active in the work with patients, the need to follow the latest trends, the need to better help clients, the need to have faster results, the need to work in a controlled setting, the need to please superiors, and so forth. None of these possible answers should be considered as right or wrong. They are influenced by our life experience and they do influence our work, our choice of concepts, and theories.

A major challenge for therapies in the next decades might be to integrate intersubjective factors in efficacy studies such as RCT studies. This may be difficult and expensive to do, and in this case, we may have to accept that RCT studies present a global figure that will remain uncompleted.

More studies are necessary to understand how to personalize treatments for a better outcome, particularly in complex cases. If we consider that EMDR is also a relational process between two unique human beings, each of them with his unique history, strengths, and weaknesses, then integrating transference and countertransference in EMDR therapy is imperative.

\section{References}

Ainsworth, M. D. S., Blehar, M., Waters, E., \& Wall, S. (1978). Patterns of attachment: A psychological study of the Strange Situation. Hillsdale, NJ: Erlbaum.

American Psychiatric Association. (2013). Diagnostic and statistical manual of mental disorders (5th ed.). Arlington, VA: American Psychiatric Publishing.

Bakermans-Kranenburg, M. J., \& van IJzendoorn, M. H. (2009). The first 10,000 adult attachment interviews: Distributions of adult attachment representations in clinical and non-clinical groups. Attachment \& Human Development, 11(3), 223-263. http: / / dx.doi.org/10.1080/ 14616730902814762

Bateman, A. W., \& Fonagy, P. (2004). Psychotherapy for borderline personality disorder: Mentalization-based treatment. New York, NY: Oxford University Press.

Bernstein, E. M., \& Putnam, F. W, Putman, F. W. (1986). Development, reliability, and validity of a dissociation scale. The Journal of Nervous and Mental Disease, 174(12), 727-735.

Brillon, P. (2005). Comment aider les victimes souffrant de stress post-traumatique. Guide l'intention des thérapeutes. Outremont, Québec: Les Editions Quebecor.

Clarkin, J. F., Yeomans, F. E., \& Kernberg, O. F. (1999). Psychotherapy for borderline personality. New York, NY: Wiley \& Sons.

Cloitre, M., Courtois, C. A., Ford, J. D., Green, B. L., Alexander, P., Briere, J., \& Van der Hart, O. (2012). The ISTSS Expert Consensus Treatment Guidelines for Complex PTSD in Adults. Retrieved from http:/ / www.istss.org/ treating-trauma/istss-complex-ptsd-treatment-guidelines.aspx

Dozier, M., Stovall-McClough, K. C., \& Albus, K. E. (2008). Attachment and psychopathology in adult-hood. In J. Cassidy \& P. R. Shaver (Eds.), Handbook of attachment: Theory, research and clinical applications (2nd ed., pp. 718744). New York, NY: Guilford Press.

Dutra, L., Bureau, J. F., Holmes, B., Lyubchik, A., \& LyonsRuth, K. (2009). Quality of early care and childhood trauma: A prospective study of developmental pathways to dissociation. The Journal of Nervous and Mental Disease, 197(6), 383-390. http://dx.doi.org/10.1097/ NMD.0b013e3181a653b7 
Dworkin, M. (2005). EMDR and the relational imperative: The therapeutic relationship in EMDR treatment. New York, NY: Routledge.

Dworkin, M., \& Errebo, N. (2010). Rupture and repair in the EMDR client/clinician relationship: Now moments and moments of meeting. Journal of EMDR Practice and Research, 4(3), 113-123.

Elkaïm, M. (2008). The use of resonance in supervision and training. Human Systems, 19, 16-25.

Farina, B., Speranza, A. M., Dittoni, S., Gnoni, V., Trentini, C., Vergano, C. M., . . .Della Marca, G. (2013). Memories of attachment hamper EEG cortical connectivity in dissociative patients. European Archives of Psychiatry and Clinical Neuroscience, 264(5), 526-458. http: / / dx.doi.org/ 10.1007/ s00406-013-0461-9

Ferenczi, S. (1916). Contributions to psychoanalysis (pp. 3080). Boston, MA: Richard G. Badger. (Original work published 1909).

Ferenczi, S. (1949). Confusion of the tongues between the adults and the children. The International Journal of Psycho-Analysis, 30, 225-230.

Ferenczi, S. (1988). Clinical diary. Cambridge, MA: Harvard University Press.

Freud, S. (1910). The future prospects of psycho-analytic therapy. Collected Papers, 2:289 (Vol. 1946). London, UK: Hogarth Press.

Gallese, V. (2009). Mirror neurons, embodied simulation, and the neural basis for social identification. Psychoanalytic Dialogues, 19(5), 519-536. http://dx.doi.org/10. $1080 / 10481880903231910$

Gallese, V., Eagle, M. N., \& Migone, P. (2007). Intentional attunement: Mirror neurons and the neural underpinnings of interpersonal relations. Journal of the American Psychoanalytic Association, 55(1), 131-175. http:/ / dx.doi. org/10.1177/00030651070550010601

Gazzola, V., Aziz-Zadeh, L., \& Keysers, C. (2006). Empathy and the somatotopic auditory mirror system in humans. Current Biology, 16(18), 1824-1829. http:/ / dx.doi.org/10. $1016 /$ j.cub.2006.07.072

Gelso, C., \& Hayes, J. (1998). The psychotherapy relationship. New York, NY: Wiley.

George, C., Kaplan, N., \& Main, M. (1996). Adult attachment interview protocol (3rd ed.). Berkeley: University of California.

Hayes, J. A. (2004). The inner world of the psychotherapist: A program of research on countertransference. Psychotherapy Research, 14(1), 21-36. http:// dx.doi.org/ $10.1093 / \mathrm{ptr} / \mathrm{kph} 002$

Heiman, P. (1950). On countertransference. International Journal of Psychoanalysis, 31, 81-84.

Hickok, G. (2009). Eight problems for the mirror neuron theory of action understanding in monkeys and humans. Journal of Cognitive Neuroscience, 21(7), 12291243. http: / / dx.doi.org/10.1162/jocn.2009.21189

International Society for the Study of Trauma and Dissociation. (2011). Guidelines for treating dissociative identity disorder in adults, third revision. Journal of Trauma
\& Dissociation: The Official Journal of the International Society for the Study of Dissociation, 12(2), 115-187.

Karpman, S. (1968). Fairy tales and script drama analysis. Transactional Analysis Bulletin, 26(7), 39-43.

Kohler, E., Keysers, C., Umiltà, M. A., Fogassi, L., Gallese, V., \& Rizzolatti, G. (2002). Hearing sounds, understanding actions: Action representation in mirror neurons. Science, 297(5582), 846-848. http:/ / dx.doi.org/ $10.1126 /$ science. 1070311

Levy, K. N. (2005). The implications of attachment theory and research for understanding borderline personality disorder. Development and Psychopathology, 17(4), 959986. http: / / dx.doi.org/10.1017/S0954579405050455

Linehan, M. M. (1993). Cognitive-behavioral treatment of borderline personality disorder. New York, NY: Guilford Press.

Liotti, G. (2006). A model of dissociation based on attachment theory and research. Journal of Trauma and Dissociation, 7(4), 55-73. http://dx.doi.org/10.1300/ J229v07n04_04

Liotti, G. (2011). Attachment disorganization and the controlling strategies: An illustration of the contributions of attachment theory to developmental psychopathology and to psychotherapy integration. Journal of Psychotherapy Integration, 21(3), 232-252. http:/ / dx.doi. org/10.1037/a0025422

Liotti, G. (2013). Phobias of attachment-related inner states in the psychotherapy of adult survivors of childhood complex trauma. Journal of Clinical Psychology, 69(11), 1136-1147. http: / / dx.doi.org/10.1002/jclp.22041

Liotti, G. (2014). L'attachement desorganisé et ses séquelles développementales dans la genèse et le traitement de l'ESPT complexe. (Disorganized attachment and its developmental sequalleas in the genesis and the treatment of complex PTSD.) Handout of the workshop given in Lausanne, Switzerland.

Liotti, G., \& Gumley, A. (2008). An attachment perspective on schizophrenia: The role of disorganized attachment, dissociation and mentalization. In $\mathrm{A}$. Moskowitz, I. Schäfer, \& M. J. Dorahy (Eds.), Psychosis, trauma and dissociation (pp. 117-133). Chichester, UK: Wiley-Blackwell.

Lyons-Ruth, K. (2003). Dissociation and the parent-infant dialogue: A longitudinal perspective from attachment research. Journal of the American Psychoanalytic Association, 51(3), 883-911. http://dx.doi.org/10.1177/ 00030651030510031501

Lyons-Ruth, K., Dutra, L., Schuder, M. R., \& Bianchi, I. (2006). From infant attachment disorganization to adult dissociation: Relational adaptations or traumatic experiences? Psychiatric Clinics of North America, 29(1), 63-86. http:/ / dx. doi.org/10.1016/j.psc.2005.10.011

Lyons-Ruth, K., \& Jacobvitz, D. (2008). Attachment disorganization: Genetic factors, parenting contexts and developmental transformation from infancy to adulthood. In J. Cassidy \& P. Shaver (Eds.), Handbook of attachment (2nd ed., pp. 667-697). New York, NY: Guilford Press. 
Main, M., \& Solomon, J. (1990). Procedures for identifying infants as disorganized/disoriented during the Ainsworth Strange Situation. In M. T. Greenberg, D. Cicchetti, \& E. M. Cummings (Eds.), Attachment in the preschool years (pp. 121-160). Chicago, IL: Chicago University Press.

Nijenhuis, E. R. S. (2017). The trinity of trauma: Ignorance, fragility, and control. The evolving concept of trauma / the concept and facts of dissociation in trauma. Göttingen, Germany: Vandenhoeck \& Ruprecht.

Panksepp, J. (1998). Affective neuroscience: The foundations of human and animal emotions. New York, NY: Oxford University Press.

Pearlman, L. A., \& Saakvitne, K. W. (1995). Trauma and the therapist: Counter-transference and vicarious traumatization in psychotherapy with incest survivors. New York, NY: W.W. Norton \& Co.

Piedfort-Marin, O., Wisler, D., Spagnoli, D., \& Piot, M. -E. (2017). An adapted version of Kluft's dimensions of therapeutic movement instrument (DTMI). European Journal of Trauma and Dissociation, 1(4), 263-268. http: / / dx.doi.org/ $10.1016 /$ j.ejtd.2017.06.006

Piedfort-Marin, O., \& Reddemann, L. (2016). Psychothérapie des traumatismes complexes: Une approche intégrative basée sur la théorie des états du Moi et des techniques hypno-imaginatives. Bruxelles: Satas.

Pizzamiglio, L., Aprile, T., Spitoni, G., Pitzalis, S., Bates, E., D’Amico, S., \& Di Russo, F. (2005). Separate neural systems for processing action- or non-action-related sounds. NeuroImage, 24(3), 852-861. http:/ / dx.doi.org/10. 1016/j.neuroimage.2004.09.025

Racker, H. (1957). The meanings and uses of countertransference. Psychoanalitical Quaterly, 26, 303-357.

Racker, H. (1968). Transference and countertransference. London, UK: The Hogarth Press.

Reddemann, L. (2011). Psychodynamisch Imaginative Trauma Therapie-PITT, das Manual (11 th edn). Stuttgart: Klett-cota.

Riggs, S. A., Paulson, A., Tunnell, E., Sahl, G., Atkison, H., \& Ross, C. A. (2007). Attachment, personality, and psychopathology among adult inpatients: Self-reported romantic attachment style versus adult attachment interview states of mind. Development and Psychopathology, 19(1), 263-291. http: / / dx.doi.org/10.1017/S0954579407070149

Rizzolatti, G., Fadiga, L., Gallese, V., \& Fogassi, L. (1996). Premotor cortex and the recognition of motor actions. Cognitive Brain Research, 3(2), 131-141. http:// dx.doi.org/ 10.1016/0926-6410(95)00038-0

Roques, J. (2004). EMDR. Une révolution thérapeutique. Paris, France: La Méridienne, Desclée de Brouwer.

Schmahl, C., Lanius, R. A., Pain, C., \& Vermetten, E. (2010). Biological framework for traumatic dissociation related to early life trauma. In R. A. Lanius, E. Vermetten, \& C. Pain (Eds.), The impact of early relational trauma on health and disease. The hidden epidemic (pp. 178-188). Cambridge, UK: Cambridge University Press.

Schore, A. (2009). Attachment trauma and the developing right brain: Origins of pathological dissociation. In P. F. Dell \& J. A. O’Neil (Eds.), Dissociation and the dissociative disorders: DSM-V and beyond (pp. 107-141). New York, NY: Routledge.

Schubbe, O. (2011). Self-care during the EMDR session: The application of the standard protocol for working with counter-transference. 12th EMDR Europe Association Conference, Vienna, Austria.

Shapiro, F. (2001). Eye movement desensitization and reprocessing: Basic principles, protocols, and procedures (2nd ed.). New York, NY: Guilford Press.

Shapiro, F. (2017). Eye movement desensitization and reprocessing: Basic principles, protocols, and procedures (3rd ed.). New York, NY: Guilford Press.

Stovall-McClough, K. C., \& Cloitre, M. (2006). Unresolved attachment, PTSD, and dissociation in women with childhood abuse histories. Journal of Consulting and Clinical Psychology, 74(2), 219-228. http://dx.doi.org/10.1037/ 0022-006X.74.2.219

Van der Hart, O., Nijenhuis, E. R. S., \& Steele, K. (2006). The Haunted Self. Structural dissociation and the treatment of chronic traumatization. New York, NY: Norton.

van Ijzendoorn, M., \& Bakermans-Kranenburg, M. J. (2008). The distribution of adult attachment representations in clinical groups: A meta-analytic search for patterns of attachment in 105 AAI studies. In H. Steele \& M. Steele (Eds.), Clinical applications of the adult attachment interview (pp. 69-96). New York, NY: Guilford Press.

Watkins, J. G., \& Watkins, H. H. (1997). Ego states theory and therapy. New York: Norton.

Wilson, M., \& Knoblich, G. (2005). The case for motor involvement in perceiving conspecifics. Psychological Bulletin, 131(3), 460-473. http:/ / dx.doi.org/10.1037/00332909.131.3.460

Young, J. E., Koslo, J. S., \& Weishaar, M. E. (2003). Schema therapy: A practitioner's guide. New York, NY: Guilford Press.

Acknowledgments. The author wishes to thank the numerous colleagues who supported this work and motivated him to write this article.

Correspondence regarding this article should be directed to Olivier Piedfort-Marin, MSc, University of Lorraine, Metz, France, APEMAC/EPSAM, EA 4360. E-mail: olivier.piedfort@gmail.com 